\title{
Strange matter and kaon to pion ratio in SU(3) PNJL model
}

\section{A. V. Friesen*}

Joint Institute for Nuclear Research, Dubna, 141980, Russia

E-mail: avfriesenetheor.jinr.ru

\section{Yu. L. Kalinovsky}

Joint Institute for Nuclear Research, Dubna, 141980, Russia

\section{D. Toneev}

Joint Institute for Nuclear Research, Dubna, 141980, Russia

\begin{abstract}
The sharp peak in the $K^{+} / \pi^{+}$ratio in relativistic heavy-ion collision is discussed. We used the SU(3) PNJL model to show how the splitting of kaon mass can appear in dense matter and how it can affect the kaon to pion ratio. In the model we calculated the $K / \pi$ ratio of both positive and negative charged mesons along the chiral phase transition line to show how the peak position can depend on the phase diagram.
\end{abstract}

Corfu Summer Institute 2018 "School and Workshops on Elementary Particle Physics and Gravity" (CORFU2018)

31 August - 28 September, 2018

Corfu, Greece

${ }^{*}$ Speaker. 


\section{Introduction}

First a sharp peak in the $K^{+} / \pi^{+}$ratio was presented by the NA49 Collaboration [1,2] and later the picture was extended with the results of other collaborations (STAR, AGS) $[3,4,5,6]$. Now it is clear that the peak appears in the ratio of positive charged mesons in heavy-ion collisions when a centre-of-mass energy is around $10 \mathrm{GeV}$. The $K^{-} / \pi^{-}$ratio shows a continuous rise without a peak. The $K^{+} / \pi^{+}$ratio is also smooth (see the Fig.1) in proton-proton collision.

The most popular theoretical description of the "horn" showed that to reproduce the peak, the theory should imply a phase transition. There is a list of most successful theoretical models:

(a) Good reproduction of the peak was obtained in the statistical model which includes both hadron resonances and $\sigma$ - meson. In this model, the critical temperature for hadrons appears in a natural way and it simulates the hadron phase transition [7].

(b) It was supposed in the SMES (the Statistical Model of Early Stages) that a jump in the ratio is a result of the deconfinement transition. It was shown that after the deconfinement transition in the strange sector $\left(m_{s} \rightarrow m_{s 0}\right)$ the strangeness yield does not depend on energy in the QGP . As a result, the $K / \pi$ ratio demonstrates smooth behaviour after jumping down [8].

(c) The microscopic transport model reproduced the experimental results after involving of the partial restoration of the chiral symmetry at the early stages of collision $[9,10,11]$. The authors showed the partial chiral symmetry restoration to be responsible for a quick increase in the $K^{+} / \pi^{+}$ratio at low energies. they explained its decrease after the maxima as a result of the chiral condensate destruction and QGP formation.

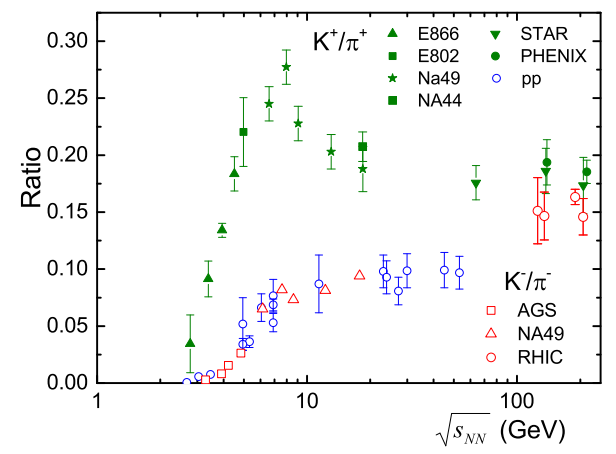

Figure 1: Left panel: the $K^{+} / \pi^{+}, K^{-} / \pi^{-}$ratio as a function of $\sqrt{s_{N N}}$ for the $\mathrm{Pb}-\mathrm{Pb}$ and $\mathrm{Au}-\mathrm{Au}$ collisions, blue circles are the $K^{+} / \pi^{+}$for pp collision.

In dense matter the creation of the $s \bar{s}$ pair dominates because of the Pauli principle: high concentration of light quarks leads to that the Fermi energy for light quarks becomes higher than the $s \bar{s}$-mass and the creation of the last one is energy-efficient. Therefore, when in the heavy ion collision the quark-gluon plasma is created, the enhancement of the strangeness can be expected in comparison with the $\mathrm{p}-\mathrm{p}$ or $\mathrm{p}-\mathrm{N}$ collision. The dependence of the ratio on the size of the system was discussed in Ref. [10, 12].

The SU(3) Nambu-Jona-Lasinio model and its Polyakov loop extension are capable of describing both the chiral phase transition, deconfinement transition and meson properties in a hot 
and dense medium $[13,14,15]$. The chiral symmetry restores when the dynamically generated quark masses drop as a function of temperature and chemical potentials. At that time, the binding energy for the pseudo-scalar meson bound states decreases and mesons dissociate changing their character to resonances with a finite lifetime (Mott effect). At low density mesons in the NJL/PNJL model are degenerated. With the density increasing splitting between kaons and antikaons occurs (as well for pions in the case when u-, d- quark masses are not equal) $[16,17,18]$. This could explain the difference in the ratios of positive and negative charged mesons.

We address this paper to the problem of the kaon to pion ratio in the context of the SU(3) PNJL model. In Sec.II, the formalism of the PNJL model and phase diagram structure will be discussed. The behavior of mesons and quarks at zero and finite density will be discussed in Sec. III. In Sec.IV, the obtained results will be discussed.

\section{SU(3) PNJL model and phase diagram}

The PNJL model is a practical tool for description of the nuclear matter at finite T and $\mu_{B}$. The Lagrangian of the model with three flavours and $U_{A}(1)$ anomaly has the form $[13,14,15]$ :

$$
\begin{aligned}
\mathscr{L} & =\bar{q}\left(i \gamma^{\mu} D_{\mu}-\hat{m}-\gamma_{0} \mu\right) q+\frac{1}{2} g_{S} \sum_{a=0}^{8}\left[\left(\bar{q} \lambda^{a} q\right)^{2}+\left(\bar{q} i \gamma_{5} \lambda^{a} q\right)^{2}\right] \\
& +g_{D}\left\{\operatorname{det}\left[\bar{q}\left(1+\gamma_{5}\right) q\right]+\operatorname{det}\left[\bar{q}\left(1-\gamma_{5}\right) q\right]\right\}-\mathscr{U}(\Phi, \bar{\Phi} ; T),
\end{aligned}
$$

where $q=(u, d, s)$ is the quark field with three flavours, $N_{f}=3$, and three colors, $N_{c}=3, \lambda^{a}$ are the Gell-Mann matrices, $a=0,1, \ldots, 8, \lambda^{0}=\sqrt{\frac{2}{3}} \mathbf{I}$ and $\mathrm{D}_{\mu}=\partial^{\mu}-i A^{\mu}$, where $A^{\mu}$ is the gauge field with $A^{0}=-i A_{4}$ and $A^{\mu}(x)=g_{S} A_{a}^{\mu} \frac{\lambda_{a}}{2}$ absorbs the strong interaction coupling. The global $\mathrm{SU}(3) \otimes \mathrm{SU}(3)$ chiral symmetry of the Lagrangian is obviously broken by introduction of the nonzero current quark masses $\hat{m}=\operatorname{diag}\left(m_{u}, m_{d}, m_{s}\right)$, and the confinement/deconfinement properties $\left(Z_{3}\right.$-symmetry) are described by the effective potential $\mathscr{U}(\Phi, \bar{\Phi} ; T)$, which is constructed on the basis of the Lattice inputs in the pure gauge sector (see for details $[15,19]$ ).

$$
\begin{aligned}
& \frac{\mathscr{U}(\Phi, \bar{\Phi} ; T)}{T^{4}}=-\frac{b_{2}(T)}{2} \bar{\Phi} \Phi-\frac{b_{3}}{6}\left(\Phi^{3}+\bar{\Phi}^{3}\right)+\frac{b_{4}}{4}(\bar{\Phi} \Phi)^{2} \\
& b_{2}(T)=a_{0}+a_{1}\left(\frac{T_{0}}{T}\right)+a_{2}\left(\frac{T_{0}}{T}\right)^{2}+a_{3}\left(\frac{T_{0}}{T}\right)^{3} .
\end{aligned}
$$

For the effective potential the following parameters were chosen: $T_{0}=0.19 \mathrm{GeV}, a_{0}=6.75, a_{1}=$ $-1.95, a_{2}=2.625, a_{3}=-7.44, b_{3}=0.75, b_{4}=7.5[20]$.

The grand potential density $\Omega\left(T, \mu_{i}\right)$ in the mean-field approximation can be obtained from the Lagrangian density (2.1) and leads to the gap equation:

$$
m_{i}=m_{0 i}-2 g_{S}\left\langle\bar{q}_{i} q_{i}\right\rangle-2 g_{D}\left\langle\bar{q}_{j} q_{j}\right\rangle\left\langle\bar{q}_{k} q_{k}\right\rangle,
$$

where $i, j, k=\mathrm{u}, \mathrm{d}, \mathrm{s}$ are chosen in cyclic order, $m_{i}$ are the constituent quark masses and the quark condensates are given by:

$$
\left\langle\bar{q}_{i} q_{i}\right\rangle=i \int \frac{d p}{(2 \pi)^{4}} \operatorname{TrS}\left(\mathrm{p}_{\mathrm{i}}\right)=-2 \mathrm{~N}_{\mathrm{c}} \int \frac{\mathrm{d}^{3} \mathrm{p}}{(2 \pi)^{3}} \frac{\mathrm{m}_{\mathrm{i}}}{\mathrm{E}_{\mathrm{i}}}\left(1-\mathrm{f}_{\Phi}^{+}\left(\mathrm{E}_{\mathrm{i}}\right)-\mathrm{f}_{\Phi}^{-}\left(\mathrm{E}_{\mathrm{i}}\right)\right)
$$


with the modified Fermi functions $f_{\Phi}^{ \pm}\left(E_{i}\right)$ :

$$
\begin{aligned}
& f_{\Phi}^{+}\left(E_{i}-\mu_{i}\right)=\frac{\bar{\Phi} e^{-\beta\left(E_{i}-\mu_{i}\right)}+2 \Phi e^{-2 \beta\left(E_{i}-\mu_{i}\right)}+e^{-3 \beta\left(E_{i}-\mu_{i}\right)}}{1+3\left(\bar{\Phi}+\Phi e^{-\beta\left(E_{i}-\mu_{i}\right)}\right) e^{-\beta\left(E_{i}-\mu_{i}\right)}+e^{-3 \beta\left(E_{i}-\mu_{i}\right)}}, \\
& f_{\Phi}^{-}\left(E_{i}+\mu_{i}\right)=\frac{\Phi e^{-\beta\left(E_{i}+\mu_{i}\right)}+2 \bar{\Phi} e^{-2 \beta\left(E_{i}+\mu_{i}\right)}+e^{-3 \beta\left(E_{i}+\mu_{i}\right)}}{1+3\left(\Phi+\bar{\Phi} e^{-\beta\left(E_{i}+\mu_{i}\right)}\right) e^{-\beta\left(E_{i}+\mu_{i}\right)}+e^{-3 \beta\left(E_{i}+\mu_{i}\right)}}
\end{aligned}
$$

where $E_{i}=\sqrt{p^{2}+m_{i}^{2}}$ is quark energy. So we take the temperature $T$, and the chemical potential of the i-quark $\left(\mu_{i}\right)$ as full independent state variables.

The model has a set of parameters: the current quark masses $m_{0 u}=m_{0 d}=5.5 \mathrm{MeV}, m_{0 s}=$ $0.131 \mathrm{GeV}$, the cut-off $\Lambda=0.652 \mathrm{GeV}$, couplings $g_{D}=89.9 \mathrm{GeV}^{-} 2$ and $g_{S}=4.3 \mathrm{GeV}^{-5}$. To use SU(3) NJL-like models, the strange quark chemical potential has to be defined. As a rule, the chemical potential of the strange quark is supposed equal to zero $\mu_{s}=0 \mathrm{GeV}$, which is ineffective for the $K / \pi$ calculations. We consider the matter with $\mu_{u}=\mu_{d}$, and $\mu_{s}=0.5 \mu_{u}$ (Case I).

Now we can discuss the phase transitions in hot and dense matter. The phase diagram in the PNJL model coincides with the common ideas of the QCD phase diagram: at high temperature and low density (chemical potential) the phase transition is soft (crossover). The phase transition is defined as a local maximum of $\left.\frac{\partial\langle\bar{q} q\rangle}{\partial T}\right|_{\mu_{B}=\text { const }}$. The deconfinement transition is determined as maximum of $\left.\frac{\partial \Phi}{\partial T}\right|_{\mu=\text { const }}$. At low temperatures and high chemical potential the crossover turns into the 1st order transition which can be defined as maximum of $\left.\frac{\partial^{2} \Omega}{\partial \mu_{u}^{2}}\right|_{T=\text { const }}[21]$. The first order phase transition ends at the point called the critical endpoint (CEP) $\left(\mu_{B, C E P}=0.972, T_{C E P}=0.11\right)$ for Case I. The critical temperature of the crossover transition at $\mu_{B}=0 \mathrm{GeV}$ is higher $\left(T_{c}=0.218\right)$ than it was predicted by the Lattice QCD $T_{c}=154(9)$ [22] and higher then experimental data for freeze-out.

As a rule, the coupling strengths $g_{S}, g_{D}$ are constant with $T, \mu$ in PNJL model. The reduction of $g_{S}$ is the way of modelling the $Z_{3}$ symmetry breaking. In $[23,24]$ it was supposed that the reason of high $T_{c}$ in the PNJL is the weak entanglement between gluons and quarks given only by $\mathrm{D}_{\mu}=\partial^{\mu}-i A^{\mu}$. As the PNJL model includes extended $Z_{3}$-symmetry [23], it is possible to introduce a phenomenological dependence of $g_{S}$ on the Polyakov loop to increase the quark-gluon coupling:

$$
\tilde{g_{S}}(\Phi)=g_{S}\left(1-\alpha_{1} \Phi \bar{\Phi}-\alpha_{2}\left(\Phi^{3}+\bar{\Phi}^{3}\right)\right)
$$

where the parameters $\alpha_{1}=\alpha_{2}=0.2$ and $T_{0}=0.19 \mathrm{GeV}$ are chosen to reproduce the LQCD data $[23,25]$.

The reduction of $g_{D}$ as a function $\mathrm{T}, \mu$ is the way of modelling the axial anomaly effect. It was mentioned in some works [26] that to explain the small size of the first order transition region in the PNJL model, the value of $g_{D}$ in medium has to differ from $g_{D_{0}}$ fixed in vacuum. For discussion of the axial anomaly, effect of $g_{D}(\mathrm{~T})$ on $\eta^{\prime}$ mass, CEP position, mixing angles and so on, we refer to the works $[26,27,28]$, and in this work we discuss only the phase diagram. Following [28], we introduce $g_{D}=g_{D_{0}} \exp -\left(\rho / \rho_{0}\right)^{2}$ where $\rho$ is the baryon density $\rho=\left(\rho_{u}+\rho_{d}+\rho_{s}\right) / 3$ with

$$
\rho_{i}=2 N_{c} \int \frac{d^{3} p}{(2 \pi)^{3}}\left(f_{\Phi}^{+}\left(E_{i}\right)-f_{\Phi}^{-}\left(E_{i}\right)\right)
$$



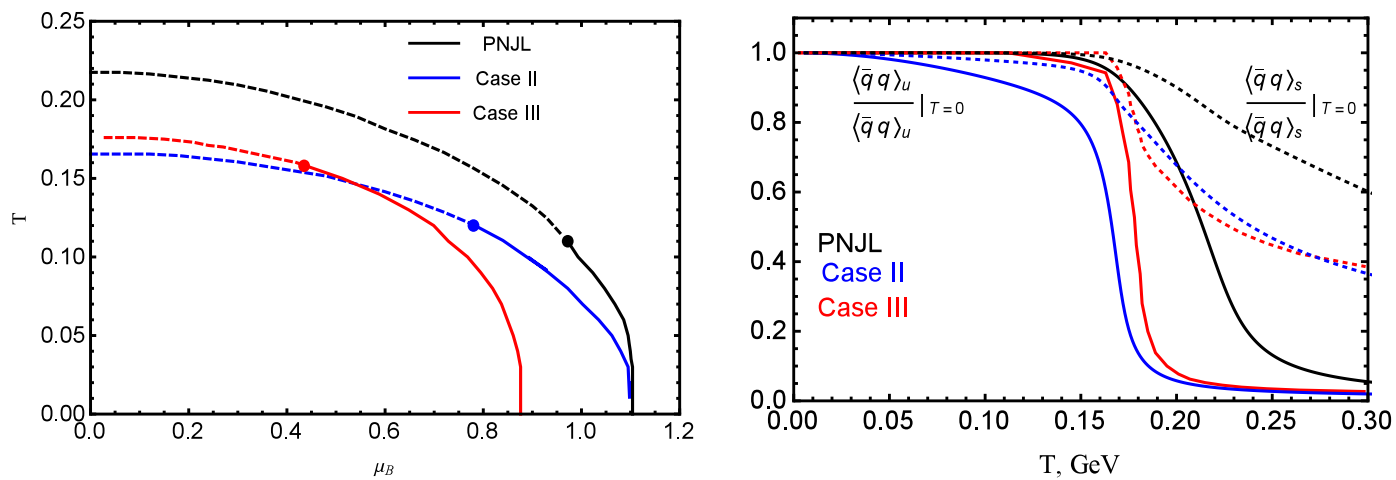

Figure 2: The phase diagrams in the T- $\mu_{B}$ plane (left panel) and the normalized condensates of light and strange quarks (right panel) for Case I (black), Case II (blue) and Case III (red).

We considered the phase diagram for the PNJL model with $\mu_{s}=0.5 \mu_{u}$ (Case I), the model with entanglement $g_{S}(\Phi)$ and $\mu_{s}=0.5 \mu_{u}$ (Case II), and we combined $g_{S}(\Phi)$ (2.6) with $g_{D}(\rho)$ (2.7) with $\mu_{s}=0.5 \mu_{u}$ (Case III). All these phase diagrams are shown in the Fig.2, left panel. The right panel of Fig. 2 demonstrates the normalised condensates for light and strange quarks. Due to the entanglement of the quark-gluon coupling in Case II, the phase transition temperature at the zero chemical potential significantly decreases. In Case III, the phase diagram is closer to the experimental one than Case I and Case II. The PNJL model is characterized by the absence of the chiral symmetry restoration for a strange quark (when the quark mass (condensate) stays large up to relatively high temperatures), see, for example, [28], nevertheless in Cases II, III a fast decrease in the strange quark condensate can be seen.

\section{Mesons in dense matter}

The meson masses are defined by the Bethe-Salpeter equation at $\mathbf{P}=0$

$$
1-P_{i j} \Pi_{i j}^{P}\left(P_{0}=M, \mathbf{P}=\mathbf{0}\right)=0,
$$

where for non-diagonal pseudo-scalar mesons $\pi, K$ :

$$
\begin{aligned}
& P_{\pi}=g_{S}+g_{D}\left\langle\bar{q}_{s} q_{s}\right\rangle, \\
& P_{K}=g_{S}+g_{D}\left\langle\bar{q}_{u} q_{u}\right\rangle .
\end{aligned}
$$

and the polarization operator has the form

$$
\Pi_{i j}^{P}\left(P_{0}\right)=4\left(\left(I_{1}^{i}+I_{1}^{j}\right)-\left[P_{0}^{2}-\left(m_{i}-m_{j}\right)^{2}\right] I_{2}^{i j}\left(P_{0}\right)\right) .
$$

Integrals $I_{1}^{i}$ and $I_{2}^{i j}\left(P_{0}\right)$ are defined as:

$$
\begin{gathered}
I_{1}^{i}=i N_{c} \int \frac{d^{4} p}{(2 \pi)^{4}} \frac{1}{p^{2}-m_{i}^{2}}=\frac{N_{c}}{4 \pi^{2}} \int_{0}^{\Lambda} \frac{\mathrm{p}^{2} d \mathrm{p}}{E_{i}}, \\
I_{2}^{i j}\left(P_{0}\right)=i N_{c} \int \frac{d^{4} p}{(2 \pi)^{4}} \frac{1}{\left(p^{2}-m_{i}^{2}\right)\left(\left(p+P_{0}\right)^{2}-m_{j}^{2}\right)} .
\end{gathered}
$$


When the meson mass exceeds the mass of its consistent $\left(P_{0}>m_{i}+m_{j}\right)$, the meson turns into the resonance state and Mott transition occurs. In this case, the complex properties of the integrals have to be taken into account and the solution has to be defined in the form $P_{0}=M_{M}-\frac{1}{2} i \Gamma_{M}$. Equation (3.1) splits into two equations from which the meson mass $M_{M}$ and the meson width $\Gamma_{M}$ can be obtained. The mass spectra for the zero chemical potential $\left(\mu_{u}=0\right)$ are shown in Fig. 3, left panel. It is clear that charged multiplets are degenerated and scalar mesons in the PNJL model decay at close temperatures $\left(T_{\text {Mott }}^{\pi}=0.232, T_{\text {Mott }}^{K}=0.23 \mathrm{Gev}\right)$.
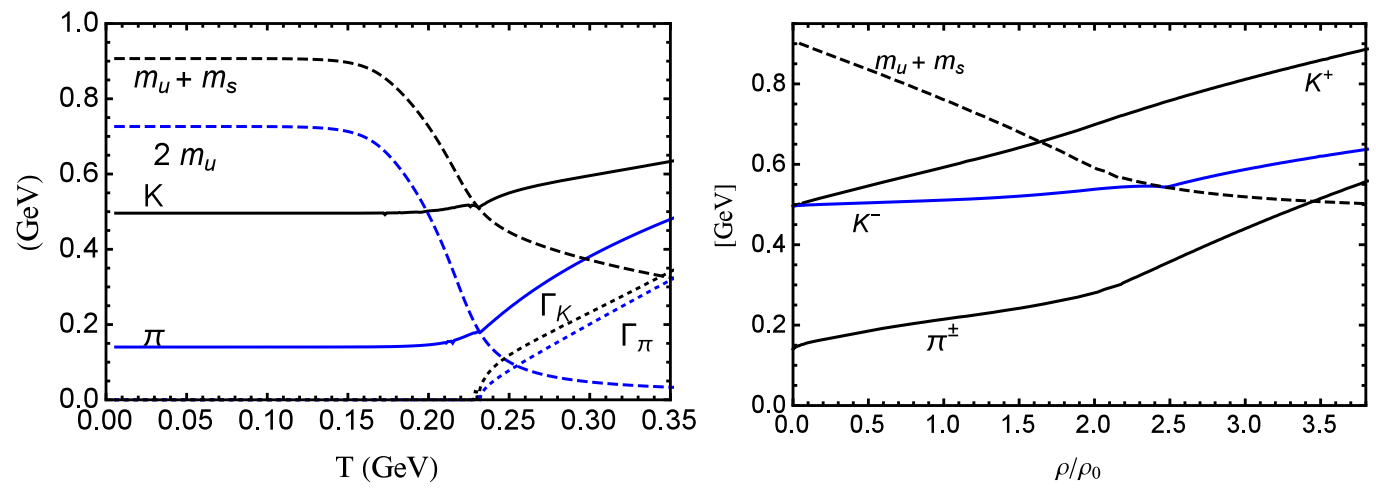

Figure 3: The spectra of meson masses as a function of the temperature at $\mu_{B}=0$ (left) and as a function of the normalized baryon density at $T=0 \mathrm{GeV}$ (right).

Though at the zero chemical potential the pions and the kaons are degenerated, their masses split with increasing density $[29,18]$. At the nonzero chemical potential and low T, the splitting of mass in charged multiplets is due to excitation of the Dirac sea modified by the presence of the medium (see Fig. 3, right panel). In dense baryon matter the concentration of light quarks is very high [30]. Therefore, the creation of a $s \bar{s}$ pair dominates because of the Pauli principle: when the Fermi energy for light quarks is higher than the $s \bar{s}$-mass, the creation of the last one is energyefficient. The increase in the $K^{+}(\bar{u} s)$ mass, with respect to that of $K^{-}(\bar{s} u)$, is justified again by the Pauli blocking for s-quark (see for discussion $[29,16]$ ). Technically, to describe the mesons in dense matter, it is needed to relate the chemical potential of quarks with the Fermi momentum $\lambda_{i}$, $\mu_{i}=\sqrt{\lambda_{i}^{2}+m_{i}^{2}}$. The pions for the chosen cases are degenerate as masses of light quarks are equal $\left(m_{u}=m_{d}\right)$.

All experimental data for the $K / \pi$ ratio were obtained in midrapidity. For the effective models, it means that the ratio of the particle number can be calculated in terms of the ratio of the number densities of mesons $\left(K^{ \pm} / \pi^{ \pm}=n_{K^{ \pm}} / n_{\pi^{ \pm}}\right)$:

$$
\begin{aligned}
& n_{K^{ \pm}}=\int_{0}^{\infty} p^{2} d p \frac{1}{e^{\left(\sqrt{p^{2}+m_{K^{ \pm}}} \mp \mu_{K^{ \pm}}\right)}-1}, \\
& n_{\pi^{ \pm}}=\int_{0}^{\infty} p^{2} d p \frac{1}{e^{\left(\sqrt{p^{2}+m_{\pi^{ \pm}}} \mp \mu_{\pi^{ \pm}}\right)}-1} .
\end{aligned}
$$

The chemical potential for pions is a phenomenological parameter and in this work it was chosen as a constant $\mu_{\pi}=0.135 \mathrm{GeV}$, following the works $[31,32,33]$. The chemical potential for kaons can be defined (see for example $[33,34]$ ) from $\mu_{q}=B_{q} \mu_{B}+S_{q} \mu_{s}+I_{q} \mu_{q}$, and in the isospin symmetry case $\left(I_{q}=0\right)$, the result is $\mu_{K}=\mu_{u}-\mu_{s}$. 

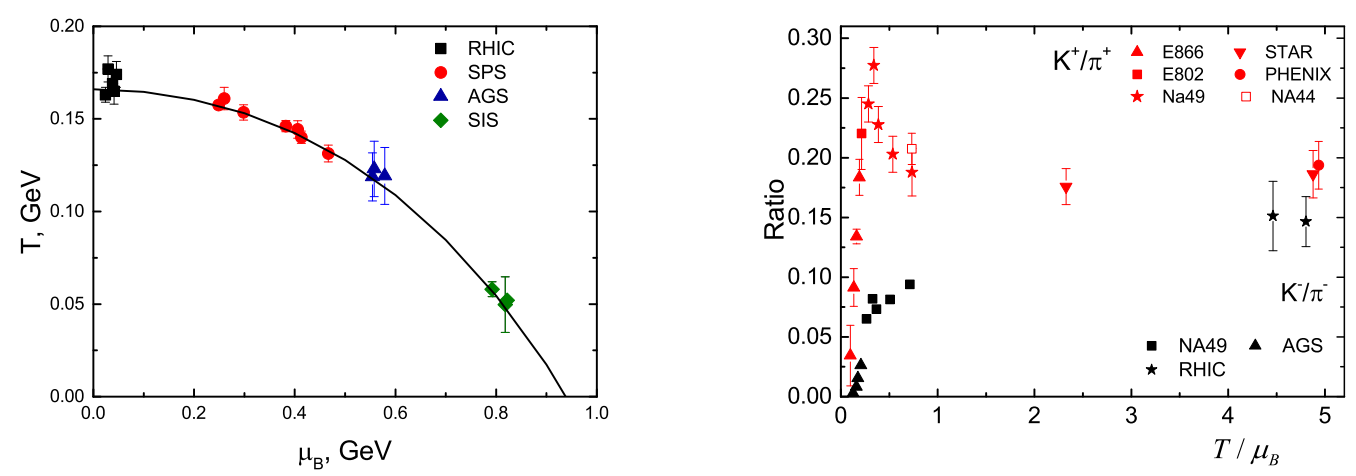

Figure 4: Left panel: the $K^{+} / \pi^{+}, K^{-} / \pi^{-}$ratio as a function of $\sqrt{s_{N N}}$ for $\mathrm{Pb}-\mathrm{Pb}$ and $\mathrm{Au}-\mathrm{Au}$ collisions, blue circles are the $K^{+} / \pi^{+}$for pp collision.

We should make some remarks to our calculations. First is that the PNJL model does not describe the dynamics of the collision of heavy ions and it can be said that its action ends at the moment of freeze-out. It makes impossible to take into account mesons created at the latest stage of collision appearing in the experiment; so the effective model results pretend only for qualitative reproduction of the data. The second is that in the effective model the collision energy $s_{N N}$ never appears as a parameter. To avoid this, we used the fact that in the statistical model for each experimental energy of collision the temperature and the baryon chemical potential of freezeout can be found using parametrization suggested, for exapmle, by J. Cleymans (see Fig.5, left panel) [35]:

$$
\begin{aligned}
T\left(\mu_{B}\right) & =a-b \mu_{B}^{2}-c \mu_{B}^{4}, \\
\mu_{B}(\sqrt{s}) & =\frac{d}{1+e \sqrt{s}},
\end{aligned}
$$

where $a=0.166 \pm 0.002 \mathrm{GeV}, b=0.139 \pm 0.016 \mathrm{GeV}^{-1}$, and $c=0.053 \pm 0.021 \mathrm{GeV}^{-3}, d=$ $1.308 \pm 0.028 \mathrm{GeV}, e=0.273 \pm 0.008 \mathrm{GeV}^{-1}$.

Using this fact, the $K / \pi$ ratio can be considered as a function of a new variable $T / \mu_{B}$ instead of $s_{N N}$, where (T, $\left.\mu_{B}\right)$ are taken along the freeze-out line (the result of rescaling is shown in Fig.5, right panel). The chemical freeze-out can be considered as a line which divides the phase where hadrons are still surviving and the phase without hadron degrees of freedom. The third assumption was to decide which of two temperatures would correspond to freeze-out T in the PNJL-approach: the temperature of the chiral phase transition or the Mott temperature. Most likely that in the mean field approach the chiral phase transition line can play the role of freeze-out as in the PNJL model the chiral phase transition line divides the hadron phase and the quark-gluon phase.

The calculated ratios $n_{K^{ \pm}} / n_{\pi^{ \pm}}$are shown in Fig.5 (left panel) as a function of the scaling variable $T / \mu_{B}$, where values $T$ and $\mu_{B}$ were chosen along the chiral phase transition line. It is clearly seen from the figure that in the region of high temperature and low density (high values of the $T / \mu_{B}$ ), the $K^{+} / \pi^{+}$and $K^{-} / \pi^{-}$-ratios tend to the same value. These results are in agreement with experimental results. In the PNJL model at high temperature and low density the difference 
between the mass of charged kaon multiplets decreases, their masses become equal to each other (kaons are degenerate at $\mathrm{T}=0$ ) and as can be seen, the difference in the ratios can also decrease.

The absence of the "horn" structure in the $K^{-} / \pi^{-}$-ratio is explained by the differnt sensibility of positive and negative charged kaons to the medium density. When the relative number of baryons decreases with increasing energy, the number of negative charged kaons does not change. The number of positive charged kaons must be balanced by strange baryons and, therefore, will decrease [36].
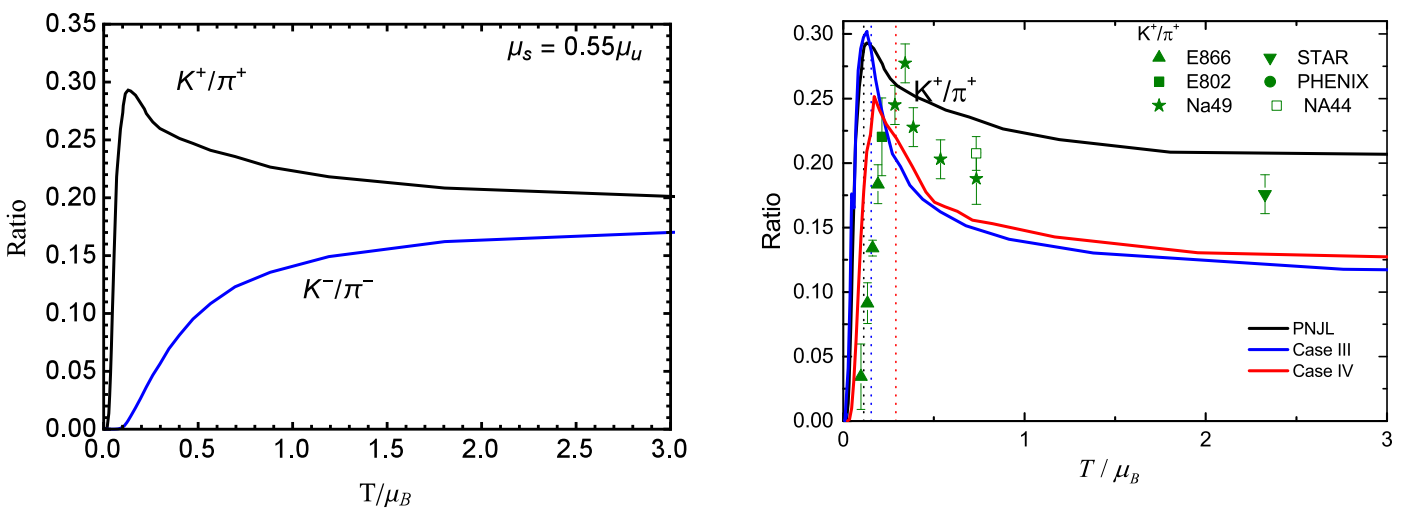

Figure 5: Left panel: the $K^{+} / \pi^{+}$and $K^{-} / \pi^{-}$ratios as functions of rescaled variable $T / \mu_{B}$. Right panel: the $K^{+} / \pi^{+}$ratio for Cases I,II, III and rescaled experimental data.

Data for the $K^{+} / \pi^{+}$ratio for three various phase diagrams are shown in Fig.5 together with the rescaled experimental data.

\section{Conclusion}

In this work, we have described the quark-meson matter in the framework of the SU(3) PNJL model. We paid special attention to the fact that the models able to describe the "horn" implied the phase transitions. In the work [10], the quick increase in the $K^{+} / \pi^{+}$ratio and its decrease with further increasing energy was interpreted as a sequence of the chiral symmetry restoration effect and the deconfinement effect. The main idea of this work was to show that the reason of the appearance of the "horn" at energies $\sqrt{s_{N N}}=8-10 \mathrm{GeV}$ may be a qualitative change in the state of the environment where kaons and pions are created.

If we assume that the detected particles can "remember" the properties of matter where they were created (the matter with the restored chiral symmetry or the deconfined matter), we can use the effective models to model the matter properties. In the PNJL model the picture is the following: when $T$ and $\mu_{B}$ are chosen along the phase diagram line, the system is in the phase transition region and the chiral condensate is still not destroyed. The main difference between the choice of $\left(T, \mu_{B}\right)$ along the line is whether we are in the crossover region or in the 1st order transition region. In the region of the 1st order transition (low temperatures) the value of $\Phi \rightarrow 0$ and the matter is confined. The values $T / \mu_{B}$ in the CEP are shown as the vertical lines in Fig.5, right panel. As can be seen, the ratio reaches the maximal value near the critical point in the region of the 1 st order transition. 
In the PNJL model, the masses of positive and negative mesons are splitted at high densities, the splitting decreases with density. This splitting can explain the difference in the behaviour of the $K / \pi$ ratios for different charges in the high-density region and the fact that they tend to the same value at high temperatures (low densities), where kaons become degenerated.

The quark matter properties can be formed by the choice of the medium scenario (a medium with an equal baryon density, a medium with beta equilibrium, strange matter, or a medium with an equal number of protons of neutrons and hyperons). In the work [37], it was illustrated that the peak position depends on the choice of the strange chemical potential: in the case, when $\mu_{u}=\mu_{d}=\mu_{s}$ ( $\mu_{K}=0$ and $\mu_{S}=0$ ) there was no enhancement in the ratio. It was also shown that the value of $\mu_{s} / \mu_{u}$ affects the position and the high of the peak in the kaon-to-pion ratio.

Generally speaking, none of these situations can reproduce the medium in a real collision of heavy ions. To match the experimental condition, the strangeness chemical potential is usually fixed to enforce the strangeness neutrality. For the PNJL model the strangeness neutrality can be introduced by additional condition $n_{s}=\frac{\partial \Omega}{\partial \mu_{s}}=0$. In the work [38], it was shown that the strangeness neutrality affected the phase diagram structure only at high $\mathrm{T}$ (or high energies) and, therefore, must not give a strong effect on the "horn".

\section{Acknowledgements}

We thank W. Cassing and D. Blaschke for the discussion. The work A.F. was supported by the Russian Science Foundation under grant no. 17-12-01427.

\section{References}

[1] S. V. Afanasiev et al. [NA49 Collabration], Phys. Rev. C 66, 054902 (2002).

[2] C. Alt,et al (NA49 Collaboration), Phys. Rev. C 77, 024903 (2008).

[3] STAR BES White paper, SN0598.

[4] J. L. Klay et al. (E895 Collaboration), Phys. Rev. C 68, 054905 (2003).

[5] B. I. Abelev et al. (STAR Collaboration), Phys. Rev. C 81, 024911. (2010); M. M. Aggarwal et al. (STAR Collaboration), ibid. 83, 024901 (2011).

[6] C. Adler et al. [STAR Collaboration], Phys. Lett. B 595 (2004) 143; arXiv: 0206008.

[7] A. Andronic, P. Braun-Munzinger, J. Stachel, Phys. Let. B 673, 142-145 (2009).

[8] M. Gazdzicki, M.I. Gorenstein, Acta Phys. Pol. B 30, 2705 (1999) [arXiv:hep-ph/9803462]; 42 (2011) 307.

[9] W. Cassing and E. L. Bratkovskaya, Phys. Rep. 308, 65 (1999).

[10] A. Palmese, W. Cassing, F. Seifer, T. Steinert, P. Moreau and E.L. Bratkovskaya, Phys. Rev. C 94, 044912 (2016).

[11] T. D. Cohen, R. J. Furnstahl and D. K. Griegel, Phys. Rev. C 45, 1881 (1992).

[12] H. Oeschler, J. Cleymans, K. Redlich, S. Wheaton, PoS CPOD2009:032,2009; arXiv:1603.09553

[13] K. Fukushima, Phys. Rev. D 77, 114028 (2008). 
[14] P. Costa, M. C. Ruivo, C. A. de Sousa, H. Hansen and W. M. Alberico Phys. Rev. D 79, 116003 (2009).

[15] E. Blanquier J. Phys. G: Nucl. Part. Phys. 38, 105003 (2011).

[16] M.C. Ruivo, C.A. de Sousa, Physics Letters B 38 (1996) 39-44

[17] P. Costa, M. C. Ruivo, Yu. L. Kalinovsky, Phys. Lett. B560 (2003) 171 - 177.

[18] P. Costa, M. C. Ruivo, Yu. L. Kalinovsky, C. A. de Sousa, Phys. Rev. C70 (2004) 025204.

[19] A. V. Friesen, Yu. L. Kalinovsky, V. D. Toneev, Int. J. Mod. Phys. A 27, 1250013, (2012); 30, 1550089 (2015).

[20] C. Ratti, M. A. Thaler, and W. Weise, Phys. Rev. D 73, 014019 (2006); arXiv:nucl-th/0604025

[21] T. Kunihiro, Phys. Lett. B 219, 363 (1989).

[22] A. Bazavov et al. (HotQCD Collaboration), Phys. Rev. D 85, 054503 (2012).

[23] Y. Sakai, T. Sasaki, H. Kuono and M. Yahiro, J. Phys. G 39, 035004 (2012); Phys. Rev. D 82, 076003 (2010).

[24] M. C. Ruivo, P. Costa and C. A. de Suosa, Phys. Rev. D 86, 116007 (2012).

[25] A. V. Friesen, Yu. L. Kalinovsky, V. D. Toneev, Physics - Uspekhi 59367 (2016).

[26] K. Fukushima Phys. Rev. 77, 114028 (2008).

[27] N. Bratovic Axial anomaly and Vector interaction in the PNJL model PhD thesis (2012).

[28] M. C. Ruivo, P. Costa and C. A. de Suosa, Phys. Rev. D 71, 116002 (2005).

[29] M. Lutz, A. Steiner, W. Weise, Nucl. Phys. A, 574755 (1994).

[30] J. Stachel, G. R. Young, Rev. Nucl. Part. Sci. 42537 (1992).

[31] M. Kataja, P.V. Ruuskanen Phys. Let. B 243, 181 (1990).

[32] V. Begun, W. Florkowski, M. Rybczynski, Phys. Rev. C 90, 014906 (2014).

[33] M. Naskret, D. Blaschke, A. Dubinin, Phys. Elem. Part. Atom. Nucl. 46, 1445 (2015).

[34] A. Lavagno and D. Pigato, EPJ Web of Conferences 37, 09022 (2012)

[35] J. Cleymans, H. Oeschler, K. Redlich, S. Wheaton, Phys. Rev. C 73, 034905 (2006)

[36] J. Cleymans, H. Oeschler, K. Redlich, S. Wheaton, arXiv:hep-ph/0504065;

[37] A. V. Friesen, Yu. L. Kalinovsky, V. D. Toneev Phys. Rev. C 99, 045201 (2019)

[38] K. Fukushima, Phys.Rev. D 79, 074015 (2009). 